\title{
Acidentes de trabalho não fatais: diferenças de gênero e tipo de contrato de trabalho
}

\author{
Non-fatal occupational injuries: \\ gender and job contract differences
}

\author{
Vilma Santana 1 \\ Antônio P. Maia 1 \\ Cláudia Carvalho 1 \\ Glaura Luz 1
}

\footnotetext{
1 Instituto de Saúde Coletiva, Universidade Federal da Bahia. Rua Padre Feijó 29, Salvador, $B A$ 40110-170, Brasil. vilma@ufba.br
}

\begin{abstract}
In Brazil, workers without a formal job contract comprise more than half of the labor force, a contingent formed mainly by women. This study presents estimates of the annual incidence of non-fatal work-related injuries and their distribution by gender and type of job contract in an urban area of Brazil. This was a community-based study with a random cluster area sample of the inhabitants from the city of Salvador, capital of the State of Bahia. The study population included all individuals from 18 to 65 years of age who reported having a paid job $(n=$ 2,947). Data were obtained through individual household interviews. The overall estimated annual incidence rate was $5.80 \%$, with a non-statistically significant difference between men (6.05\%) and women (5.53\%) or workers with (5.67\%) and without (5.92\%) a formal job contract. These similarities between work-related accidents across gender and type of job contract highlighted the need for greater attention to this problem among women and informal workers, who are largely ignored in official statistics.
\end{abstract}

Key words Occupational Accidents; Occupational Health; Contracts; Gender

Resumo No Brasil, trabalhadores sem vínculo formal de trabalho representam mais da metade da força de trabalho, cuja maioria é composta por mulheres. Neste estudo, estima-se a incidência anual de acidentes ocupacionais não fatais, e a sua distribuição por gênero e tipo de contrato de trabalho, em uma área urbana do Brasil. Dados provêm de um inquérito de base comunitária conduzido com uma amostra aleatória dos habitantes de Salvador, capital do Estado da Bahia. A população deste estudo compreende o total de pessoas entre 18 e 65 anos que referiram trabalho remunerado $(n=2.947)$. A coleta de dados baseada em entrevistas domiciliares individuais. A incidência anual de acidentes foi estimada em 5,80\%, discretamente maior entre os homens (6,05\%) do que entre as mulheres (5,53\%), ou entre trabalhadores sem contrato formal de trabalho (5,92\%) do que no grupo de comparação (5,67\%), diferenças não estatisticamente significantes. A semelhança entre as freqüências de acidentes de trabalho entre mulheres e homens independentemente do tipo de contrato de trabalho apontam para a necessidade de maior atenção a estes eventos no sexo feminino, e entre os trabalhadores informais, amplamente ignorados nas estatísticas oficiais do país.

Palavras-chave Acidentes de Trabalho; Saúde Ocupacional; Contrato; Gênero 


\section{Introdução}

Acidentes de trabalho se constituem em problema de saúde pública em todo o mundo, por serem potencialmente fatais, incapacitantes e acometerem, em especial, pessoas jovens e em idade produtiva, o que acarreta grandes conseqüências sociais e econômicas. No Brasil, a importância dos acidentes de trabalho se expressa pelo custo total estimado de $\mathrm{R} \$ 12,5$ bilhões por ano para as empresas, e de cerca de $\mathrm{R} \$ 20$ bilhões por ano para o país (Pastore, 1999), ainda que estes dados sejam restritos a empresas registradas e seus trabalhadores contratados contribuintes da Previdência Social. Para o ano de 1997, o Ministério do Trabalho e Emprego estimou que 2.802 trabalhadores perderam suas vidas em acidentes de trabalho, e que cerca de 11.152 pessoas receberam benefícios por incapacidade permanente (MTE, 2000). Apesar de suas conseqüências negativas, acidentes de trabalho ressentem-se de uma insuficiente atenção por parte das políticas sociais no país. Evidência disso é a pobre qualidade dos dados oficiais sobre a mortalidade e morbidade por acidentes de trabalho, reconhecidamente subestimadas, tanto por inadequações do sistema de registro, quanto pela parcialidade da cobertura, ainda restrita aos trabalhadores com carteira de trabalho (Wünsch Filho, 1999). Portanto, inexistem dados oficiais sobre a morbidade por acidentes de trabalho no Brasil para todos os trabalhadores, desde que os dados oficiais limitam-se aos trabalhadores do setor formal da economia ou aos que possuem contratos de trabalho registrados.

No entanto, o crescimento do número de trabalhadores sem contrato formal de trabalho no país tem sido significativo, chegando a representar a maioria da força de trabalho em algumas regiões urbanas (Cacciamali, 1997). A maioria das pessoas que compõe este segmento da força de trabalho é formada por mulheres, que também apresentam outras diferenças ocupacionais em relação aos homens (Biddle \& Blanciforti, 1999). Estudos conduzidos em outros países têm mostrado que trabalhadores temporários (Quinlan et al., 2001; Silverstein et al., 1998), terceirizados (François \& Lievin, 1995), ou do setor informal (Loewenson, 1998) estão envolvidos em atividades mais perigosas como as de manutenção, ou são realizadas sem as medidas de proteção requeridas para os demais trabalhadores (Quinlan et al., 2001). Estes, entre outros aspectos, colocam tal grupo de trabalhadores em maior risco para acidentes e outras enfermidades ocupacionais (Quinlan et al., 2001). Embora as estatísticas apon- tem que as mulheres têm menor risco para acidentes de trabalho, fatais ou não fatais, do que os homens (Biddle \& Blanciforti, 1999), um adequado dimensionamento do problema, levando em conta a experiência ocupacional historicamente distinta das mulheres ainda está por ser realizado. É possível que mudanças no perfil ocupacional do sexo feminino que vêm ocorrendo em todo o mundo, decorrentes de uma maior participação de mulheres em atividades consideradas perigosas, possam estar determinando alterações na morbidade ou mortalidade por acidentes ocupacionais (Quinlan et al., 2001).

Neste estudo, com base em dados coletados em um inquérito populacional, estima-se a incidência anual de acidentes ocupacionais não fatais, para os trabalhadores com e sem contrato de trabalho, analisando-se homens e mulheres separadamente. A distribuição da incidência dos acidentes segundo variáveis demográficas, sociais e ocupacionais é apresentada com vistas a subsidiar o planejamento das ações de prevenção, e também a identificação de hipóteses para análises posteriores. Para tal, os dados são apresentados de modo desagregado por gênero e tipo de contrato de trabalho.

\section{Métodos}

Os dados deste estudo provêm de um inquérito de base comunitária realizado em Salvador, capital do Estado da Bahia, Nordeste do Brasil. Estimou-se em cerca de 2,7 milhões os moradores de Salvador, terceira maior concentração urbana do país, que detinha uma das maiores taxas nacionais de desemprego, de trabalhadores sem carteira assinada, e de pessoas vivendo em favelas da periferia (IBGE, 1999). A população do estudo compõe-se de uma amostra dos moradores desta região, que foram entrevistados entre agosto/dezembro de 2000. Neste estudo específico, devido às peculiaridades da atividade ocupacional e dos acidentes de trabalho entre adolescentes e crianças, restringese a análise aos trabalhadores remunerados de idade entre 18 e 65 anos.

A amostra deste estudo foi do tipo aleatória por conglomerados de estágio único, tomando-se como referência subáreas da região urbana de Salvador. Empregaram-se mapas disponibilizados pela Companhia de Desenvolvimento da Região Metropolitana de Salvador (CONDER), existentes para toda a região. Subáreas de mapas na escala 1:12.000 foram numeradas e sorteadas. Para cada uma delas, foram obtidos mapas em escala 1:2.000, que per- 
mitiam tanto a visualização de construções e detalhes topográficos, quanto melhores condições para identificação dos limites das subáreas da amostra. Visitas foram então realizadas a cada uma dessas subáreas para contato com organizações comunitárias e lideranças, quando então se identificavam pontos de referência e novas construções residenciais. Com estes dados se elaboravam croquis em computador, utilizados para auxiliar na localização das residências pelos entrevistadores de campo. O número de subáreas a ser sorteado foi estimado com base no tamanho amostral pretendido, na média de pessoas na faixa de idade de interesse por cada família $(3,8)$ (IBGE, 1999), e no número médio destas $(n=86,6)$ por subárea. Das 32 subáreas necessárias que foram sorteadas, três não eram habitadas e, portanto, foram descartadas, restando 29, nas quais todas as pessoas das famílias residentes no domicílio foram identificadas para a coleta de dados.

A escolha desse desenho amostral foi motivada especialmente pelas características urbanas dessa região metropolitana, onde é comum a falta ou imprecisão de endereços residenciais. Outra razão foi a perspectiva comunitária do estudo, que prevê o estudo de redes de apoio social, vizinhança e também a participação de grupos e associações comunitárias, para facilitar operacionalmente a pesquisa e também permitir a devolução do conhecimento produzido. Além disso, esta estratégia permitiu uma permanência dos pesquisadores de campo por maior tempo no mesmo local, o que possibilitou melhor integração com a população, o reconhecimento dos pesquisadores pelos moradores e, conseqüentemente, maior segurança, especialmente nas áreas consideradas perigosas. Este desenho também permitiu uma coleta de dados mais rápida por causa da proximidade geográfica das famílias selecionadas e um menor número de perdas no seguimento pretendido da população, por se tratar da fase basal de um estudo de coorte.

Cada uma das famílias foi inicialmente registrada, após obtenção de consentimento de participação para a pesquisa. Em seguida à identificação das famílias, aprazavam-se entrevistas individuais com os indivíduos elegíveis, aos quais, individualmente, se apresentava a pesquisa e solicitava-se o consentimento individual. A coleta de dados foi realizada por entrevistadores treinados, supervisionados por estagiários e professores envolvidos com a pesquisa. Na primeira visita, após a apresentação dos objetivos da pesquisa e a aquisição do consentimento de participação, foi realizado um censo da família, registrando-se todos os seus membros, independentemente da idade. Nesta etapa, identificavam-se as pessoas que tinham atividade remunerada ou realizavam trabalho doméstico na própria casa, para a própria família. Para estas pessoas, eram então agendadas entrevistas pessoais, para a coleta de outros dados sócio-demográficos, ocupacionais, e também sobre a ocorrência de acidentes nos doze meses anteriores à entrevista. A checagem e correção de informações foi feita por meio de re-entrevistas pessoais ou telefônicas. Os instrumentos de pesquisa foram questionários desenvolvidos especificamente para o estudo, cujo conteúdo foi definido com base num mapa conceitual desenvolvido pela equipe, para identificação de aspectos mensuráveis a serem incluídos nos instrumentos de pesquisa. Isso foi realizado com base na literatura existente, nos modelos teóricos e nas próprias experiências pessoais das equipes de epidemiologia e etnografia envolvidas no projeto, além de instrumentos empregados em outras investigações (Barata et al., 2000; Fachini et al., 1998). A linguagem e factibilidade operacional dos instrumentos foram testadas em um estudo piloto.

Considerou-se como acidentes de trabalho qualquer dano infligido ao corpo por transferência de energia durante o trabalho, ou deslocamento até o local do trabalho que envolvesse uma curta duração entre exposição e efeitos identificáveis após a ocorrência do evento/circunstância (Hagberg et al., 1997). Como se previa que a percepção e reconhecimento de acidentes ocupacionais por trabalhadores do setor informal estivesse prejudicada pela predominância de uma visão previdenciária, demarcada por propósitos administrativos e compensatórios empregados pelo sistema nacional de seguridade social, voltada para os trabalhadores contratados formalmente, decidiu-se por uma abordagem indireta. Ou seja, perguntavase inicialmente "você sofreu algum acidente, de qualquer natureza, alguma vez na vida?”; e em seguida, especificava-se o período referente aos últimos doze meses. Em caso positivo, pedia-se ao respondente para especificar as circunstâncias em que este havia ocorrido. Quando o acidente havia ocorrido durante a realização de atividades de trabalho, ou durante o trajeto para o trabalho, passava-se a um questionário específico, no qual se registrava uma breve narrativa do evento, empregando-se as próprias palavras do entrevistado. Desse modo, não se formulava nenhuma pergunta direta sobre acidente de trabalho, ficando a inferência sobre a ocorrência de acidente de trabalho sob a responsabilidade do entrevistador, que foi treinado para esta tarefa, baseando-se nas cir- 
cunstâncias descritas. Quando havia referência a mais de um acidente no período de referência do estudo, considerava-se apenas o mais recente. Todas as ocupações no período foram registradas, sendo que a principal era identificada pelo próprio entrevistado, ao qual se solicitava que empregasse os critérios de maior remuneração ou maior proporção de tempo alocado. Os dados sobre ocupação foram registrados em aberto para posterior codificação. A existência ou não de contrato formal de trabalho foi analisada observando-se o registro em carteira de trabalho. Devido às peculiaridades do vínculo contratual, excluíram-se os funcionários públicos, empresários, e profissionais liberais. Outras variáveis de interesse foram a cor da pele, identificada pelo entrevistador e classificada em negros e não negros; o nível de escolaridade categorizado em: (baixo) analfabetos e aqueles com o 1 o grau incompleto que equivale a menos de 8 anos de escolaridade; (médio) com o 1o e ou 2 o graus completos; (alto) com curso superior completo ou incompleto. O nível sócio-econômico foi definido com base no número de bens da família ou equipamentos pertencentes à família, especificamente: automóvel, computador, máquina de lavar, vídeo cassete, toca-disco a laser, microondas, máquina de lavar louça, telefone e casa de praia. O número total de itens foi categorizado em: (baixo) menos de 3 itens; (médio) 3 a 5 itens; (alto) acima de 5 itens.

A incidência anual foi calculada dividindose o número de casos identificados de acidentes de trabalho não fatais, no período de referência, pelo total da população do estudo. Note que, embora o estudo seja transversal, o fato de acidentes serem eventos circunscritos no tempo permite a estimativa de casos incidentes, com base em informações referidas no passado. Dados de ocupações desenvolvidas simultaneamente ou consecutivamente, no ano de referência da pesquisa, foram registrados, mas nesta análise leva-se em conta apenas a ocupação principal atual no momento da entrevista. Apresentam-se a incidência bruta de acidentes não fatais geral e específicas para categorias das variáveis descritoras. Testes estatísticos para diferenças de freqüências foram o Qui Quadrado de Pearson, não se definindo um único alfa dada a natureza descritiva do estudo. A construção da base de dados foi feita com dupla digitação realizada por pessoas diferentes, utilizando-se o Epi Info-6.0, o que possibilitou que as bases fossem comparadas e então erros pudessem ser detectados e corrigidos. A análise dos dados foi realizada com o SAS 8.11, empregando-se para o ajuste relativo ao desenho amostral, das estimativas finais, o Proc Surveymeans deste mesmo programa. O protocolo da pesquisa foi submetido e aprovado por uma Comissão de Ética em Pesquisa do Hospital Professor Edgard Santos da Universidade Federal da Bahia, tendo sido atendidas todas as recomendações legais. $\mathrm{O}$ anonimato $\mathrm{e}$ confidencialidade das informações foram mantidas nos registros dos dados e nas publicações. Toda a equipe da pesquisa foi instruída sobre os dispositivos e responsabilidades éticas do estudo.

\section{Resultados}

Das 2.512 famílias selecionadas, identificaramse 9.591 indivíduos, dos quais 3.527 eram trabalhadores remunerados, com idade entre 18 a 65 anos. Destes, 209 (5,90\%) não responderam aos questionários individuais, restando 3.318, dos quais excluíram-se $10(0,30 \%)$ cujos dados sobre vínculo de trabalho não haviam sido registrados. Além desses, em razão do interesse nas diferenças de vínculo contratual deste trabalho, excluíram-se 260 (7,80\%) funcionários públicos, 48 (1,45\%) empresários, 31 (0,93\%) profissionais liberais e $22(0,60 \%)$ para os quais faltavam dados sobre carteira de trabalho. Assim, a população deste estudo compreende 2.947 pessoas, da qual a maioria $(53,76 \%)$ era do sexo masculino e não tinha carteira de trabalho $(52,76 \%)$. A incidência anual de acidentes de trabalho não fatais foi estimada em 5,8\% (171/2.947), maior entre os homens $(6,05 \%)$ do que entre as mulheres $(5,53 \%)$, diferença não estatisticamente significante. Também não houve diferenças estatisticamente significantes na incidência estimada para os trabalhadores sem carteira de trabalho (TSC - 5,92\%) e com carteira (TCC - 5,67\%).

Na Tabela 1, apresentam-se as características sócio-demográficas da população de estudo de acordo com o gênero e tipo de contrato de trabalho. Entre as mulheres, TSC foram a maioria $(p<0,01)$ e entre os homens cerca de metade da força de trabalho não apresentava contrato de trabalho formal. Em relação à distribuição da idade, embora as TSC tenham a mesma média de idade do que as TCC (dados não apresentados), verifica-se uma predominância de mulheres nos grupos mais jovens (18-20 anos), diferença estatisticamente significante $(\mathrm{p}<0,05)$. Observa-se também que as TSC têm menor escolaridade e maior proporção de pobres do que as TCC $(\mathrm{p}<0,01)$. Entre os homens, os TSC também prevalecem no grupo mais jovem (18-20 anos), mas distinta- 
Características sócio-demográficas da população do estudo de acordo com o gênero

e tipo de contrato de trabalho da ocupação principal ( $n=2.947)$.

\begin{tabular}{|c|c|c|c|c|c|c|c|c|}
\hline \multirow[t]{3}{*}{ Variáveis } & \multicolumn{4}{|c|}{ Mulheres $(n=1.392)$} & \multicolumn{4}{|c|}{ Homens ( $n=1.555)$} \\
\hline & \multicolumn{2}{|c|}{$\begin{array}{c}\text { Sem carteira } \\
(\mathrm{n}=792 ; 56,9 \%)\end{array}$} & \multicolumn{2}{|c|}{$\begin{array}{c}\text { Com carteira } \\
\left(n=600 ; 43,1 \%^{\star \star}\right)\end{array}$} & \multicolumn{2}{|c|}{$\begin{array}{c}\text { Sem carteira } \\
(n=763 ; 49,1 \%)\end{array}$} & \multicolumn{2}{|c|}{$\begin{array}{c}\text { Com carteira } \\
(n=792 ; 50,9 \%\end{array}$} \\
\hline & $\mathrm{n}$ & $\%$ & $n$ & $\%$ & $\mathrm{n}$ & $\%$ & $\mathrm{n}$ & $\%$ \\
\hline \multicolumn{9}{|l|}{ Idade em anos } \\
\hline $18-20$ & 107 & 13,5 & 53 & 8,8 & 94 & 12,3 & 64 & 8,1 \\
\hline $21-30$ & 233 & 29,4 & 202 & 33,7 & 209 & 27,4 & 296 & 37,4 \\
\hline $31-40$ & 217 & 27,4 & 174 & 29,0 & 196 & 25,7 & 236 & 29,8 \\
\hline $41-65$ & 235 & 29,7 & 171 & 28,5 & 264 & 34,6 & 196 & 24,7 \\
\hline \multicolumn{9}{|l|}{ Situação conjugal } \\
\hline Solteiros & 342 & 43,6 & 279 & 46,7 & 292 & 38,9 & 265 & 33,9 \\
\hline Casados & 341 & 43,4 & 250 & 41,9 & 421 & 56,0 & 488 & 62,5 \\
\hline Separados/viúvos & 102 & 13,0 & 68 & 11,4 & 38 & 5,1 & 28 & 3,6 \\
\hline \multicolumn{9}{|l|}{ Cor da pele } \\
\hline Não negra & 301 & 38,0 & 234 & 39,0 & 295 & 38,7 & 318 & 40,1 \\
\hline Negra & 491 & 62,0 & 366 & 61,0 & 468 & 61,3 & 474 & 59,9 \\
\hline \multicolumn{9}{|c|}{ Nível de escolaridade } \\
\hline Baixo & 408 & 51,5 & 205 & 34,2 & 457 & 59,9 & 355 & 44,8 \\
\hline Médio & 331 & 41,8 & 342 & 57,0 & 253 & 33,2 & 387 & 48,9 \\
\hline Alto & 53 & 6,7 & 53 & 8,8 & 53 & 6,9 & 50 & 6,3 \\
\hline \multicolumn{9}{|c|}{ Nível sócio-econômico } \\
\hline Baixo & 437 & 55,2 & 272 & 45,5 & 407 & 53,6 & 398 & 50,3 \\
\hline Médio & 222 & 28,0 & 223 & 37,3 & 226 & 29,7 & 275 & 34,7 \\
\hline Alto & 133 & 16,8 & 103 & 17,2 & 127 & 16,7 & 119 & 15,0 \\
\hline
\end{tabular}

Nível de escolaridade: baixo (analfabetos ou com 1ㅇ grau incompleto); médio (com 1ㅇ ou 2o graus completos); alto (curso superior completo ou incompleto); Nível sócio-econômico, baseado no número de bens e equipamentos disponíveis no domicílio de uma lista definida: baixo (menos de 3 itens); médio (3 a 5 itens); alto (acima de 5 itens). * p-valor $<0,05$.

$\star \star p$-valor $<0,01$, referente ao $X^{2}$ de Pearson.

mente do grupo das mulheres, isto volta a ocorrer na faixa de maior idade, entre 41 e 65 anos $(\mathrm{p}<0,01)$. Ainda entre os homens, nota-se que os TSC são mais freqüentemente solteiros ou separados/viúvos e têm menor nível de escolaridade do que os TCC ( $\mathrm{p}<0,001)$, mas não houve diferenças relativas ao nível sócio-econômico. A distribuição da cor da pele de acordo com o tipo de contrato de trabalho, não apresentou diferenças tanto entre as mulheres quanto entre os homens.

Características ocupacionais segundo o sexo e tipo de contrato de trabalho são mostradas na Tabela 2. Pode-se observar que, para as mulheres, o setor que congrega a maior proporção de trabalhadores é o de serviços gerais/domésticos/limpeza com $29,7 \%$ da força de trabalho, seguido pelo comércio com $24,2 \%$ e administração/ensino com 18,8\%; enquanto para os homens, o comércio é o que responde pela maior freqüência relativa de trabalhadores, $22,8 \%$, e respectivamente, $17,8 \%$ na construção civil, $12,2 \%$ nos transportes e $8,1 \%$ na indústria. Nota-se também uma maior dispersão dos trabalhadores entre os ramos de atividade entre os homens, quando comparados às mulheres. Entre estas, TSC predominam no ramo do comércio, serviços gerais/domésticos, artesanal tradicional, e outros serviços, embora as diferenças tenham sido pequenas. Ao contrário, entre os homens, as diferenças são marcantes, especialmente no setor da construção civil em que TSC representam o dobro dos TCC; logo após aparece o ramo artesanal tradicional, comércio e outros não classificados, nessa ordem. Essas diferenças são estatistica- 
Características ocupacionais da população do estudo, de acordo com o gênero e tipo de contrato de trabalho da ocupação principal $(n=2.947)$.

\begin{tabular}{|c|c|c|c|c|c|c|c|c|}
\hline \multirow[t]{3}{*}{ Variáveis } & \multicolumn{4}{|c|}{ Mulheres $(n=1.392)$} & \multicolumn{4}{|c|}{ Homens $(n=1.555)$} \\
\hline & \multicolumn{2}{|c|}{$\begin{array}{c}\text { Sem carteira } \\
(n=792 ; 56,9 \%)\end{array}$} & \multicolumn{2}{|c|}{$\begin{array}{c}\text { Com carteira } \\
\left(\mathrm{n}=600 ; 43,1 \%^{\star \star}\right)\end{array}$} & \multicolumn{2}{|c|}{$\begin{array}{c}\text { Sem carteira } \\
(n=763 ; 49,1 \%)\end{array}$} & \multicolumn{2}{|c|}{$\begin{array}{c}\text { Com carteira } \\
(n=792 ; 50,9 \%\end{array}$} \\
\hline & $\mathrm{n}$ & $\%$ & $n$ & $\%$ & $\mathrm{n}$ & $\%$ & $\mathrm{n}$ & $\%$ \\
\hline \multicolumn{9}{|l|}{ Ramo de atividade } \\
\hline Comércio & 208 & 26,3 & 129 & 21,5 & 192 & 25,2 & 163 & 20,6 \\
\hline Serviços gerais/domésticos & 249 & 31,4 & 165 & 27,5 & 38 & 5,0 & 77 & 9,7 \\
\hline Administração/ensino & 115 & 14,5 & 146 & 24,3 & 40 & 5,2 & 64 & 8,1 \\
\hline Indústria & 5 & 0,6 & 20 & 3,3 & 40 & 5,2 & 86 & 10,9 \\
\hline Construção & 1 & 0,1 & 2 & 0,3 & 181 & 23,7 & 95 & 12,0 \\
\hline Transporte & 3 & 0,4 & 4 & 0,7 & 63 & 8,3 & 127 & 16,0 \\
\hline Artesanal & 67 & 8,5 & 16 & 2,7 & 37 & 4,9 & 3 & 0,4 \\
\hline Segurança & 4 & 0,5 & 1 & 0,2 & 14 & 1,8 & 80 & 10,1 \\
\hline Saúde & 17 & 2,2 & 51 & 8,5 & 5 & 0,7 & 9 & 1,1 \\
\hline Outros serviços & 52 & 6,6 & 28 & 4,7 & 62 & 8,1 & 57 & 7,2 \\
\hline Outros & 71 & 9,0 & 38 & 6,3 & 91 & 11,9 & 31 & 3,9 \\
\hline \multicolumn{9}{|l|}{ Perfil de atividades } \\
\hline Apenas trabalho pago & 218 & 27,5 & 255 & 42,5 & 591 & 77,5 & 657 & 83,0 \\
\hline Trabalho pago e para a família & 549 & 69,3 & 334 & 55,7 & 155 & 20,3 & 114 & 14,3 \\
\hline Dois trabalhos pagos & 17 & 2,2 & 6 & 1,0 & 14 & 1,8 & 21 & 2,7 \\
\hline Outro & 8 & 1,0 & 5 & 0,8 & 3 & 0,4 & 0 & - \\
\hline Recebeu treinamento & 269 & 34,2 & 324 & 54,5 & 364 & 48,1 & 528 & 66,8 \\
\hline \multicolumn{9}{|l|}{ Local de trabalho } \\
\hline Empresa/firma & 166 & 25,8 & 415 & 78,3 & 241 & 32,0 & 621 & 78,5 \\
\hline Repartição pública & 33 & 5,1 & 54 & 10,2 & 20 & 2,7 & 40 & 5,1 \\
\hline Na rua & 95 & 14,8 & 3 & 0,6 & 184 & 24,3 & 75 & 9,5 \\
\hline Própria casa & 176 & 27,4 & 0 & - & 88 & 11,7 & 3 & 0,4 \\
\hline Casa de outros & 102 & 15,9 & 23 & 4,3 & 130 & 17,2 & 11 & 1,4 \\
\hline Outros & 71 & 11,0 & 35 & 6,6 & 91 & 12,1 & 40 & 5,1 \\
\hline \multicolumn{9}{|l|}{ Como chega ao trabalho } \\
\hline Ônibus & 227 & 48,2 & 415 & 78,3 & 293 & 44,2 & 520 & 66,1 \\
\hline Andando & 176 & 37,4 & 53 & 10,0 & 203 & 30,6 & 79 & 10,1 \\
\hline Carro próprio & 29 & 6,2 & 25 & 4,7 & 89 & 13,4 & 67 & 8,5 \\
\hline Carro/ônibus da empresa & 6 & 1,3 & 19 & 3,6 & 29 & 4,4 & 88 & 11,2 \\
\hline Mais de um meio & 5 & 1,1 & 2 & 0,4 & 1 & 0,2 & 3 & 0,4 \\
\hline Outros & 28 & 5,8 & 16 & 3,0 & 48 & 7,2 & 29 & 3,7 \\
\hline
\end{tabular}

Subtotais diferem devido a dados não registrados; ${ }^{\star} p$-valor $<0,05 ;{ }^{* \star} p$-valor $<0,01$ referente ao $X^{2}$ de Pearson. 
mente significantes ( $\mathrm{p}<0,001)$. Quanto ao perfil de atividades de trabalho, verifica-se que enquanto TSC do sexo feminino, em maioria, acumulam trabalho pago e obrigações com atividades domésticas para a família $(69,3 \%)$, entre os homens a maior concentração é no trabalho remunerado exclusivo. Ainda nesta Tabela 2, pode-se notar que para ambos os gêneros, a proporção de trabalhadores que referiu ter recebido treinamento para o trabalho foi menor entre os TSC $(p<0,01)$. Vale ressaltar que a maior parte, tanto de mulheres quanto de homens TSC, desenvolve atividades remuneradas na própria casa, na rua, e em casa de outras pessoas $(\mathrm{p}<0,01)$, respectivamente. Enquanto o modo mais comum de locomoção para o traba- lho para ambos os sexos e qualquer tipo de contrato, importante para se compreender os acidentes de trajeto, é o uso de transporte coletivo, exemplificado pelos ônibus urbanos ( $\mathrm{p}<0,01)$.

A incidência anual de acidentes de trabalho não fatais estimada com base em dados referidos de acordo com variáveis sócio-demográficas está apresentada na Tabela 3 . Verifica-se que entre as mulheres, esta incidência foi maior entre as TSC $(6,1 \% / 4,8 \%)$, e entre os homens, ao contrário, a incidência foi maior entre os TCC $(6,3 \% / 5,8 \%)$, diferenças não estatisticamente significantes. Interessante fazer ver as distinções na distribuição dos acidentes de trabalho não fatais, segundo o gênero e tipo de contrato de trabalho, que em razão da disper-

Incidência dos acidentes de trabalho não fatais de acordo com as características sócio-demográficas, gênero e tipo de contrato de trabalho da ocupação principal ( $n=2.947)$.

\begin{tabular}{|c|c|c|c|c|c|c|c|c|}
\hline \multirow[t]{3}{*}{ Variáveis } & \multicolumn{4}{|c|}{ Mulheres $(n=1.392)$} & \multicolumn{4}{|c|}{ Homens ( $n=1.555)$} \\
\hline & \multicolumn{2}{|c|}{$\begin{array}{c}\text { Sem carteira } \\
(\mathrm{n}=792 ; \mathrm{I}=6,1 \%)\end{array}$} & \multicolumn{2}{|c|}{$\begin{array}{c}\text { Com carteira } \\
(\mathrm{n}=600 ; \mathrm{I}=4,8 \%)\end{array}$} & \multicolumn{2}{|c|}{$\begin{array}{c}\text { Sem carteira } \\
(\mathrm{n}=763 ; \mathrm{I}=5,8 \%)\end{array}$} & \multicolumn{2}{|c|}{$\begin{array}{c}\text { Com carteira } \\
(\mathrm{n}=792 ; \mathrm{I}=6,3 \%\end{array}$} \\
\hline & $\mathrm{n}$ & $\%$ & $n$ & $\%$ & $n$ & $\%$ & $n$ & $\%$ \\
\hline \multicolumn{9}{|l|}{ Idade em anos } \\
\hline $18-21$ & 107 & 7,5 & 53 & 3,8 & 94 & 5,3 & 64 & 7,8 \\
\hline $21-30$ & 233 & 6,0 & 202 & 5,0 & 209 & 8,6 & 296 & 6,8 \\
\hline $31-40$ & 217 & 5,5 & 174 & 4,0 & 196 & 4,6 & 236 & 5,5 \\
\hline $41-65$ & 235 & 6,0 & 171 & 5,9 & 264 & 4,6 & 196 & 6,1 \\
\hline \multicolumn{9}{|l|}{ Situação conjugal } \\
\hline Solteiros & 342 & 5,9 & 279 & 4,7 & 292 & 5,8 & 265 & 6,0 \\
\hline Casados & 341 & 6,5 & 250 & 5,6 & 421 & 5,7 & 488 & 5,7 \\
\hline Divorciado/separado/viúvo & 102 & 5,9 & 68 & 2,9 & 38 & 5,3 & 28 & 10,7 \\
\hline \multicolumn{9}{|l|}{ Cor da pele } \\
\hline Não negra & 301 & 3,7 & 234 & 3,4 & * 295 & 5,4 & 318 & 4,7 \\
\hline Negra & 491 & 7,5 & 366 & 5,7 & 468 & 6,0 & 474 & 7,4 \\
\hline \multicolumn{9}{|l|}{ Nível de escolaridade } \\
\hline Baixo & 408 & 6,4 & 205 & 6,8 & 457 & 7,4 & 355 & 8,2 \\
\hline Médio & 331 & 6,3 & 342 & 4,1 & 253 & 3,6 & 387 & 4,7 \\
\hline Alto & 53 & 1,9 & 53 & 1,9 & 53 & 1,9 & 50 & 6,0 \\
\hline \multicolumn{9}{|l|}{ Nível sócio-econômico } \\
\hline Baixo & 437 & 6,0 & 272 & 5,5 & 407 & 6,1 & 398 & 7,5 \\
\hline Médio & 222 & 5,9 & 223 & 4,9 & 226 & 5,3 & 275 & 5,8 \\
\hline Alto & 133 & 6,8 & 103 & 2,9 & 127 & 5,5 & 119 & 3,4 \\
\hline
\end{tabular}

I = Incidência cumulativa anual; Nível de escolaridade: baixo (analfabetos ou com 1o grau incompleto); médio (com 1ㅇ ou 2ㅇ graus completos); alto (curso superior completo ou incompleto); Nível sócio-econômico, baseado no número de bens e equipamentos disponíveis no domicílio de uma lista definida: baixo (menos de 3 itens); médio (3 a 5 itens); alto (acima de 5 itens).

* $\mathrm{p}$-valor $<0,05$.

** $\mathrm{p}$-Valor $<0,01$ referente ao $X^{2}$ de Pearson. 
são dos dados, não permite inferências estatísticas. Entre as mulheres, o risco de acidentes é cerca de duas vezes maior entre as TSC no grupo jovem de 18 a 21 anos, divorciadas/separadas/viúvas, e ou de nível sócio-econômico alto, diferenças não estatisticamente significantes (Tabela 3). Ainda entre as mulheres, verifica-se que TSC negras apresentam risco de acidentes de trabalho não fatais quase duas vezes maior do que o grupo referente $(\mathrm{p}<0,05)$. Diferença semelhante também ocorreu entre as TCC, mas não foi estatisticamente significante. Também dignos de nota são os achados de maior incidência entre as mulheres de baixa escolaridade e nível sócio-econômico, limitados às TCC. Para o sexo masculino, não se observaram diferenças marcantes na incidência de acidentes não fatais com o contrato de trabalho que mereçam consideração, dada a variabilidade aleatória. Todavia, vale advertir que a incidência no grupo de baixa escolaridade é o dobro do grupo de nível médio ( $\mathrm{p}<0,05)$, apenas nos TSC. Homens TCC, ao contrário, apresentam maior risco de acidentes de trabalho não fatal quando pertencentes aos estratos sócio-econômicos baixo ou médio, quando comparados ao superior $(\mathrm{p}<0,05)$.

$\mathrm{Na}$ Tabela 4, mostram-se as incidências anuais de acidentes de trabalho não fatais específicas para certas características do trabalho. Entre as mulheres, confirma-se que o risco de acidentes de trabalho não fatais é maior entre as TSC no ramo de comércio $(p=0,06)$. Similarmente, entre os homens, o ramo de atividade que apresenta maior diferencial de risco para acidentes, entre TSC, é o de comércio. Interessante ressaltar que o trabalho em via pública e ir andando para o trabalho parecem aumentar o risco de acidentes de trabalho não fatais; ao passo que para mulheres TCC, ter dupla jornada de trabalho e também ir andando para o trabalho elevam a morbidade por acidentes. No sexo masculino, os TSC com dupla jornada de trabalho, seja com o trabalho para a família, com duas atividades remuneradas, ou que trabalham na própria casa, ou vão andando para o trabalho, têm incidência de acidentes de trabalho aumentada em relação às demais categorias.

\section{Discussão}

Os resultados deste estudo mostram que a ocorrência de acidentes de trabalho não fatais é de grande magnitude, e suas características diferem, de modo complexo e variado, em consonância com o gênero e também em relação ao tipo de contrato de trabalho. Entretanto, não se verificaram diferenças na incidência de acidentes de trabalho não fatais de acordo com o gênero e tipo de contrato de trabalho, em níveis estatisticamente significantes. $\mathrm{O}$ achado mais importante é, sem dúvida, a alta incidência desses agravos à saúde, estimada em 5,80\% por ano, o que evidencia a sua relevância quantitativa para a saúde pública, como também a sua invisibilidade. Isto se salienta, por exemplo, em um possível sub-registro elevado das estatísticas oficiais, cujos dados apresentados, para o Estado da Bahia no ano de 1990, revelavam uma incidência anual de apenas $0,34 \%$ (Wünsch Filho, 1999). Neste estudo, a incidência anual dos acidentes de trabalho não fatais, estimada com base em informações referidas de base populacional, é consistente com a de 5,0\% relatada para o Brasil (Frumkim \& Câmara, 1991), e um pouco mais elevada do que a encontrada por Barata et al., (2000), de 4,24\%, para o Estado de S. Paulo, ainda que tenham empregado metodologia semelhante à deste estudo. Mesmo que seja conhecido que trabalhadores da indústria têm maior risco de acidentes de trabalho não fatais do que os demais ramos de atividade, foram estimadas taxas de incidência anual de 5,6/100 pessoa-ano em uma siderúrgica de Minas Gerais, muito próximas às estimadas nesta pesquisa (Schoemaker et al., 2000). Isto contrasta com os resultados encontrados em uma indústria de celulose (Fassa et al., 1996), na qual a freqüência destes agravos foi bem mais elevada (23,0\% ao ano), apesar do maior risco para acidentes observado nas indústrias (Frumkim \& Câmara, 1991). Portanto, pode-se assegurar que as estimativas deste estudo se aproximam das encontradas em pesquisas conduzidas com metodologia semelhante no Brasil, realizadas com a população geral e também com trabalhadores de indústrias.

Considerando os dados deste estudo e o apresentado por Wünsch Filho (1999), é possível calcular a proporção da subestimativa da incidência de acidentes de trabalho não fatais, em $94,13 \%$ [(5,8 - 0,34)/5,8], o que corresponderia a um coeficiente de correção para as taxas oficiais de 17,05 , assumindo-se os necessários pressupostos. Como os dados de outros países baseiam-se em registros de notificações, além de serem apresentados conjuntamente com as doenças ocupacionais, comparações ficam limitadas. Dentre os poucos resultados que permitem distinção entre esses agravos, encontra-se um estudo baseado em dados registrados em serviços de emergência nos Estados Unidos, que indica uma incidência de 
Incidência anual de acidentes de trabalho não fatais de acordo com características ocupacionais,

gênero e tipo de contrato da ocupação principal $(n=2.947)$.

\begin{tabular}{|c|c|c|c|c|c|c|c|c|}
\hline \multirow[t]{3}{*}{ Variáveis } & \multicolumn{4}{|c|}{ Mulheres $(n=1.392)$} & \multicolumn{4}{|c|}{ Homens $(n=1.555)$} \\
\hline & \multirow{2}{*}{\multicolumn{2}{|c|}{$\begin{array}{c}\text { Sem carteira } \\
(\mathrm{n}=792 ; \mid=6,1 \%)\end{array}$}} & \multirow{2}{*}{\multicolumn{2}{|c|}{$\begin{array}{c}\text { Com carteira } \\
(n=600 ; 1=4,8 \%)\end{array}$}} & \multirow{2}{*}{\multicolumn{2}{|c|}{$\begin{array}{c}\text { Sem carteira } \\
(\mathrm{n}=763 ; \mathrm{I}=5,8 \%)\end{array}$}} & \multirow{2}{*}{\multicolumn{2}{|c|}{$\begin{array}{c}\text { Com carteira } \\
(n=792 ; 1=6,3 \%)\end{array}$}} \\
\hline & & & & & & & & \\
\hline \multicolumn{9}{|l|}{ Ramo de atividade } \\
\hline Comércio & 208 & 8,2 & 129 & 3,1 & 192 & 6,8 & 163 & 3,7 \\
\hline Serviços gerais/domésticos & 249 & 8,0 & 165 & 6,1 & 38 & 2,6 & 77 & 13,0 \\
\hline Administração/ensino & 115 & 4,4 & 146 & 2,7 & 40 & 2,5 & 64 & 1,6 \\
\hline Indústria & 5 & - & 20 & 5,0 & 40 & 7,5 & 86 & 11,6 \\
\hline Construção & 1 & - & 2 & - & 181 & 6,6 & 95 & 8,4 \\
\hline Transporte & 3 & - & 4 & - & 63 & 1,6 & 127 & 4,7 \\
\hline Artesanal & 67 & 4,5 & 16 & 6,3 & 37 & 5,4 & 3 & 33,3 \\
\hline Segurança & 4 & - & 1 & - & 14 & - & 121 & 5,0 \\
\hline Saúde & 16 & 5,9 & 51 & 3,9 & 5 & - & 9 & 22,2 \\
\hline Outros serviços & 52 & 3,9 & 28 & 10,7 & 62 & 11,3 & 57 & 7,0 \\
\hline Outros & 71 & - & 38 & 10,5 & 91 & 4,4 & 31 & 3,2 \\
\hline \multicolumn{9}{|l|}{ Perfil de atividades } \\
\hline Apenas trabalho pago & 218 & 5,5 & 255 & 3,5 & 591 & 4,7 & 657 & 5,6 \\
\hline Trabalho pago e para a família & 549 & 6,4 & 334 & 6,0 & 155 & 9,0 & 114 & 9,7 \\
\hline Dois trabalhos pagos & 17 & 5,9 & 6 & - & 14 & 14,3 & 21 & 9,5 \\
\hline Outro & 8 & - & 5 & - & 3 & - & 0 & - \\
\hline \multicolumn{9}{|l|}{ Recebeu treinamento } \\
\hline Sim & & 5,2 & & 5,6 & & 6,6 & & 6,3 \\
\hline Não & & 6,4 & & 4,1 & & 4,9 & & 6,5 \\
\hline \multicolumn{9}{|l|}{ Local de trabalho } \\
\hline Empresa/Firma & 166 & 6,0 & 415 & 4,6 & 241 & 2,9 & 621 & 6,1 \\
\hline Repartição pública & 33 & 3,0 & 54 & 3,7 & 20 & 5,0 & 40 & 10,0 \\
\hline Na rua & 95 & 8,4 & 3 & - & 184 & 6,5 & 75 & 8,0 \\
\hline Própria casa & 176 & 5,1 & 0 & - & 88 & 10,2 & 3 & - \\
\hline Casa de outros & 102 & 4,9 & 23 & 4,4 & 130 & 6,2 & 11 & 9,1 \\
\hline Outros & 71 & 2,8 & 35 & 5,7 & 91 & 7,7 & 40 & 2,5 \\
\hline \multicolumn{9}{|l|}{$\begin{array}{l}\text { Meio de transporte } \\
\text { para o trabalho }\end{array}$} \\
\hline Ônibus & 227 & 5,3 & 415 & 4,3 & 293 & 3,1 & 520 & 6,4 \\
\hline Andando & 176 & 7,4 & 53 & 9,4 & 203 & 8,4 & 79 & 6,3 \\
\hline Carro próprio & 29 & 3,5 & 25 & - & 89 & 2,3 & 67 & 4,5 \\
\hline Carro/ônibus empresa & 6 & - & 19 & - & 29 & 3,5 & 88 & 4,6 \\
\hline Mais de um & 5 & - & 2 & - & 1 & - & 3 & - \\
\hline Outros & 28 & - & 16 & 6,3 & 48 & 12,5 & 29 & 17,2 \\
\hline
\end{tabular}

I = Incidência cumulativa anual

* $\mathrm{p}$-valor $<0,05$ referente ao $\mathrm{X}^{2}$ de Pearson 
2,9/100 FTE (equivalente de trabalhadores de tempo integral) entre indivíduos de idade igual ou acima de 15 anos, sendo 3,4/100 FTE no grupo do sexo masculino, e de 2,1/100 FTE para as mulheres (CDC, 2001). Um dos poucos inquéritos comunitários individuais sobre acidentes de trabalho foi conduzido no Zimbábue, embora tenha se restringido a trabalhadores do setor informal (Lowenson, 1998). Foi estimada em $13,1 \%$ a freqüência de acidentes de trabalho não fatais em um ano, achado 10 vezes maior do que os dados oficiais para os trabalhadores do setor formal neste mesmo país (Loewenson, 1998). Os contextos ocupacionais, sociais e culturais distintos, tornam difícil, porém, este tipo de comparação com outros países.

Neste estudo, os achados relativos à distribuição dos acidentes de trabalho não fatais por gênero demonstram que as diferenças são pequenas e não estatisticamente significantes. A exceção é para a cor da pele entre as mulheres sem contrato formal de trabalho, que mostra que as mulheres de cor negra têm um risco maior do que as não negras $(p<0,05)$, fato que não acontece para os homens $(\mathrm{p}>0,05)$. Entre os homens sem contrato de trabalho, é o nível de escolaridade que se associa negativamente com o risco de acidentes $(\mathrm{p}<0,05)$. É possível que os resultados de estudos que mostram os homens em maior risco de acidentes de trabalho do que as mulheres estejam, de fato, apresentando vieses de seleção por registrarem preferencialmente trabalhadores do setor formal. Ao incluir as mulheres trabalhadoras sem contrato de trabalho, essas diferenças por gênero desapareceram. Vale notar que esses resultados devem ser tomados com precaução, uma vez que é amplamente sabido que mulheres tendem a relatar mais freqüentemente morbidade ou sintomas, quando respondendo a questionários individuais, como a metodologia empregada neste estudo. A favor destes resultados, no entanto, vale frisar que narrativas detalhadas foram registradas de cada um dos acidentados, incluindo-se dados de recebimento de tratamento médico, o que reduz a possibilidade de que tenham sido superestimados em termos absolutos. Contudo, é difícil evitar as limitações na comparação da freqüência desses eventos com os homens.

Ainda em relação às diferenças de gênero, nota-se que mulheres TSC apresentam maior incidência de acidentes de trabalho não fatais do que os homens, em cada um dos ramos de atividades, apesar de se observar o contrário para as atividades artesanais tradicionais e outros serviços, nos quais os maiores riscos ocorrem entre pessoas do sexo masculino, diferen- ças não estatisticamente significantes. Também digno de nota são os maiores riscos para mulheres TSC que trabalham em empresas/firmas quando comparadas aos homens $(p=0,12)$, achado restrito aos trabalhadores sem contrato de trabalho. Quando se analisam os resultados para os TCC, verifica-se que homens se acidentam mais comumente do que as mulheres, no ramo de serviços gerais/domésticos $(\mathrm{p}<0,06)$ e de saúde $(\mathrm{p}<0,04)$. Estas diferenças podem estar sendo causadas por distinções de gênero nas tarefas desempenhadas por esses trabalhadores, ainda que no mesmo ramo de atividades.

A comparação da ocorrência de acidentes de trabalho não fatais entre trabalhadores com carteira e sem carteira mostra que também não houve diferenças significativas, apesar de esses grupos diferirem na sua composição demográfica, social e ocupacional. Encontrou-se que, entre mulheres TSC, não existem diferenças significativas na incidência de acordo com dados sócio-demográficos, à exceção do maior risco entre as de cor negra. Isto pode representar maior exposição a riscos de acidentes destes grupos resultante de acesso diferencial a ocupações menos qualificadas, e, portanto, de maior perigo para acidentes, por parte das mulhres de cor negra. Como é óbvio, os mais importantes preditores de acidentes de trabalho são ocupacionais, especialmente o ramo de atividade, ocupação e tarefas desenvolvidas. Neste estudo, verifica-se que trabalhadoras sem contrato de trabalho têm maior risco de acidentes não fatais do que as demais trabalhadoras, quando é considerado o ramo do comércio. Nos demais ramos as diferenças são pequenas ou podem ter ocorrido em conseqüência de variações aleatórias devido ao escasso número de indivíduos nos grupos em comparação.

Em suma, observou-se que, embora as diferenças de gênero e vínculo contratual de trabalho demarquem distinções sociais, demográficas e ocupacionais, não são possivelmente os mais importantes definidores de padrões de risco em geral para os acidentes, que poderão ser explorados nas especificidades das ocupações e atividades desempenhadas. Além disto, é importante também delinear uma melhor compreensão dos diferenciais relativos à cor da pele encontrados para as mulheres, e para o grau de escolaridade entre os homens, quando não dispõem de contrato formal de trabalho; e, ainda, a tendência a menores riscos para as mulheres, quando têm a carteira de trabalho assinada. Ou seja, são menores as diferenças de gênero nos trabalhadores sem contrato, e entre estes, são os aspectos relacionados à dis- 
criminação racial e nível de escolaridade que contam mais significativamente como possíveis mecanismos de compreensão desse perfil de morbidade. Tais resultados não são conclusivos dada a própria natureza exploratória deste estudo, sendo portanto necessárias análises dirigidas especificamente a estas hipóteses.

Trabalhadores sem vinculação contratual comumente estão envolvidos em atividades do setor informal, que abrangem pequenas empresas ou atividades não regulamentadas pelo Estado, como o comércio de rua ou o trabalho temporário exercidos no domicílio ou como atividade autônoma realizada por conta própria (Cacciamali, 1997). No Brasil, representam indivíduos que não dispuseram de acesso à educação ou à qualificação profissional; logo, abrigariam as ocupações menos seguras. Entretanto, os dados deste estudo não revelam cifras desfavoráveis para os trabalhadores do setor informal, seja para as mulheres ou homens, salvo para o ramo de comércio, quando considerado o sexo feminino, e o de serviços gerais/domésticos para os homens. Mas é possível que a fraca efetivação das políticas de proteção aos trabalhadores no país, tornem estes trabalhadores "menos diferentes" ou "mais parecidos", em termos dos riscos a que estão expostos, o que resultaria em menores distinções na magnitude de ocorrência desses acidentes de trabalho. De interesse para discussão é que tanto os TCC como os TSC encontram-se, em termos gerais, em alto risco de sofrerem acidentes de trabalho, o que merece igual atenção dos órgãos públicos, voltados preferencialmente para o setor formal da economia em que predominam os trabalhadores com contrato formal de trabalho. Estes, por mais que estejam sob o abrigo de políticas de proteção, podem estar se ressentindo de uma pobre efetivação da legislação trabalhista em vigor. Além disso, torna-se visível o extenso sub-registro dos acidentes e enfermidades ocupacionais que são de notificação obrigatória e a precariedade do sistema de fiscalização dos ambientes de trabalho ou a pequena adoção das normas de higiene e segurança do trabalho, já apontadas por Wünsch Filho (1999).

Em epidemiologia ocupacional, estudos de base populacional geral são tradicionalmente realizados com dados secundários obtidos de registros de notificação oficial, em que pesem as conhecidas limitações de qualidade e cobertura observadas em quase todos os países (Leigh et al., 1997; Mital et al., 1999; Wünsch Filho, 1999). Estas dificuldades são ainda maiores em regiões onde o trabalho sem registro chega a alcançar mais da metade da força de trabalho, como o Brasil, e não se dispõe de cadastramentos que permitam o acesso a denominadores populacionais confiáveis. Ademais, os agravos à saúde de origem ocupacional não são reconhecidos e/ou registrados como tais nos serviços de saúde ou nos locais de trabalho (Santana, 2002). É possível que com a efetivação das recentes políticas de proteção à saúde do trabalhador propostas para o SUS, em especial com a integração ao Programa de Saúde da Família (MS, 2001), seja possível o alcance de estimativas mais acuradas e precisas. No entanto, mudanças que se encontram em discussão, como a privatização do seguro acidente de trabalho poderão comprometer este processo.

Neste trabalho, não se testam hipóteses nem se apresentam avaliações de fatores de risco, mas sim os resultados de morbidade de acidentes não fatais e as características sociais, demográficas e ocupacionais da distribuição da incidência em consonância com o objetivo do estudo. O seu desenho, um inquérito de base populacional não é tradicional para este tipo de propósito, mas parece ser a única possibilidade viável de serem obtidas estimativas confiáveis para este importante problema de saúde pública. A morbidade baseada em dados referidos em questionários tem a sua validade questionável, pois é sujeita a erros de memorização, sobretudo para os agravos de pouca gravidade cuja lembrança depende do tempo de ocorrência. Além disso, trabalhadores do setor informal, autônomos ou biscateiros, por exemplo, podem não ter muito clara a noção de que estão sujeitos a acidentes de trabalho, um conceito amplamente previdenciário em nossa sociedade, o que levaria à omissão da informação. Por isso é possível que estes resultados tenham sido subestimados, visto que o período de referência do estudo (um ano) é prolongado, muito embora alguns cuidados tenham sido tomados para a redução desses erros. Por exemplo, a abordagem inicial foi feita considerando-se acidentes de qualquer tipo, tendo sido omitida qualquer pergunta específica sobre a sua relação ou caracterização como acidente de trabalho. Sua natureza era inferida, valendo-se do conteúdo da narrativa, pelos membros da equipe, desse modo evitando-se os limites da percepção ocupacional desses eventos por parte dos trabalhadores sem contrato de trabalho.

Estudos de base populacional também se ressentem da dispersão dos números de indivíduos em cada uma das categorias ocupacionais, o que reflete a sua distribuição na população. Entretanto, trazem como vantagem estimativas que expressam a conformação geral do 
perfil epidemiológico existente, permitindo sinalizar grupos ocupacionais que poderão ser abordados com metodologias apropriadas. $\mathrm{Pa}-$ ra complementar estes achados, estudos com base em dados secundários, empregando diferentes fontes e recursos de "captura e recaptura”, juntamente com os registros de serviços de emergência poderão contribuir para a construção de um quadro mais completo desse problema; ou ainda estudos nacionais com refe- rência aos modelos de áreas-sentinela, como vem sendo proposto pela equipe que realizou este trabalho. Os resultados deste estudo, em que pesem as suas limitações, trazem informações, particularmente sobre a real magnitude do problema dos acidentes de trabalho não fatais, de grande relevância para as pessoas envolvidas com as políticas e gestão de programas de saúde e segurança para o trabalhador.

\section{Agradecimentos}

Este trabalho somente se tornou possível com a excepcional dedicação de todos os membros da nossa extensa equipe e da população de Salvador, que nos acolheu com carinho e respeito. É a ela que dedicamos esta pesquisa, esperando que possa vir a frutificar em melhores políticas de proteção aos trabalhadores.

Fontes de financiamento: Ministério de Ciência e Tecnologia, Conselho Nacional de Desenvolvimento Científico e Tecnológico (CNPq/Proc. 521226-98-8/Projeto Nordeste de Pesquisa) e PIBIC/Universidade Federal da Bahia; Secretaria de Planejamento do Estado da Bahia, Superintendência de Apoio ao Desenvolvimento Científico e Tecnológico (CADCT) No. 68/2000.

\section{Referências}

BARATA, R. B.; RIBEIRO, M. C. \& MORAES, J. C., 2000. Acidentes de trabalho referidos por trabalhadores moradores da Região Metropolitana de São Paulo em 1994. Informe Epidemiológico do SUS, 9:199-210.

BIDDLE, E. A. \& BLANCIFORTI, L. A., 1999. Impact of a changing U.S. workforce on the occupational injury and illness experience. American Journal of Industrial Medicine, 36(Sup. 1):7-10.

CACCIAMALI, M. C., 1997. O Mercado de Trabalho no Brasil: O Aumento da Informalidade nos Anos 90. Notas sobre o Processo de Informalização no Mercado de Trabalho no Contexto da Globalização. Brasília: Instituto de Pesquisa Econômica Aplicada. (mimeo.)

CDC (Centers for Disease Control and Prevention), 2001. Nonfatal occupational injuries and illnesses treated in hospital emergency departments United States, 1998. JAMA, 285:2443-2444.

FASSA, A. G.; FACCHINI, L. A. \& DALL'AGNOL, M. M., 1996. Trabalho e mortalidade comum em indústria de celulose e papel: Um perfil segundo setor. Cadernos de Saúde Pública, 12:297-307.

FACCHINI, L. A.; DALL'AGNOL, M. M. \& FASSA, A. G., 1998. Trabalho e Saúde Infantil. Projeto de Pesquisa. Pelotas: Faculdade de Medicina, Universidade Federal de Pelotas. 
FRANÇOIS, M. \& LIEVIN, D., 1995. Emplois Precaires et Accidentabilité: Enquete Statistique dans 85 Entreprises. Lyon: Service Ergonomie et Psychologie Industrielle Section Ergonomie des Systemes.

FRUMKIM, H. \& CÂMARA, V., 1991. Occupational and health safety in Brazil. American Journal of Public Health, 81:1619-1624.

HAGBERG, M. D.; CHRISTIANI, D.; COURTNEY, T. K.; HALPERIN, W.; LEAMON, T. B. \& SMITH, T. J., 1997. Conceptual and definitional issues in occupational injury epidemiology. American Journal of Industrial Medicine, 32:106-115.

IBGE (Fundação Instituto Brasileiro de Geografia e Estatística), 1999. Pesquisa Nacional por Amostragem de Domicílios, PNAD - Síntese de Indicadores, 1998. 5 Fevereiro 2001 <http://www. ibge.net/home/estatistica/populacao/trabalhoe rendimento/pnad2001/default.shtml/ >.

LEIGH, J. P.; MARKOWITZ, J. P.; FAHS, M.; SHIN, C. \& LANDRIGAN, P. J., 1997. Occupational injury and illness in the United States: Estimates of costs, morbidity, and mortality. Archives of Internal Medicine, 157:1557-1568.

LOWENSON, R., 1998. Health impact of occupational risks in the informal sector in Zimbabwe. International Journal of Environment and Occupational Health, 4:264-274.

MITAL, A.; PENNATHUR, A. \& KANSAL, A., 1999. Non-fatal occupational injuries in the United States Part I - Overall trends and data summaries. International Journal of Industrial Ergonomics, 25:109-129.

MS (Ministério da Saúde), 2001. Plano de Ação em Saúde do Trabalhador. Brasília: Coordenação da Saúde do Trabalhador, Secretaria de Políticas em Saúde, Ministério da Saúde.
MTE (Ministério do Trabalho e Emprego), 2000. Es tatísticas sobre Acidentes de Trabalho. 3 Março $2000<$ http://www.mtb.gov.br>.

PASTORE, J., 1999. A Dimensão Econômica dos Acidentes e Doenças do Trabalho. Brasília: Serviço Social da Indústria. (mimeo.)

QUINLAN, M.; MAYHEW, C. \& BOHLE, P., 2001. The global expansion of precarious employment, work disorganizaion, and consequences for occupational health: A review of recent research. International Journal of Health Services, 31:335-413.

SANTANA, V., 2002. Algumas questões e recomendações sobre as estatísticas de acidentes de trabalho no Brasil. In: I Seminário sobre Estatísticas sobre Acidentes e Doenças do Trabalho no Brasil, Anais, pp. 75-79, São Paulo: Fundação Jorge Duprat Figueiredo de Segurança e Medicina do Trabalho, Ministério do Trabalho e Emprego.

SCHOEMAKER, M.; BARRETO, S. M.; SWERDLOW, A. J.; HIGGINS, C. D. \& CARPENTER, R. G., 2000. Non-fatal work related injuries in a cohort of Brasilian steelworkers. Occupational and Environmental Medicine, 57:555-562.

SILVERSTEIN, B.; FOLEY, M. \& SAMA, S., 1998. Protecting Contingent workeres form Work-related Injury. Helsinki: Finnish Institute of Occupational Health.

WÜNSCH FILHO, V., 1999. Reestruturação produtiva e acidentes de trabalho no Brasil: Estrutura e tendência. Cadernos de Saúde Pública, 15:41-51.

Recebido em 23 de novembro de 2001

Versão final reapresentada em 29 de agosto de 2002

Aprovado em 22 de novembro de 2002 